\title{
Infection prophylaxis and management of fungal infections in lung transplant
}

\author{
Armelle Pérez-Cortés Villalobos, Shahid Husain \\ Multi-Organ Transplant Unit, Division of Infectious Diseases, University Health Network, Toronto, ON, Canada \\ Contributions: (I) Conception and design: All authors; (II) Administrative support: None; (III) Provision of study materials or patients: None; (IV) \\ Collection and assembly of data: All authors; (V) Data analysis and interpretation: All authors; (VI) Manuscript writing: All authors; (VII) Final \\ approval of manuscript: All authors. \\ Correspondence to: Shahid Husain, MD, MS, FRCP. 585 University Avenue, 11 PMB 138, Toronto, Ontario, M5G 2N2, Canada. \\ Email: shahid.husain@uhn.ca.
}

\begin{abstract}
Lung transplantation has emerged as a lifesaving treatment for a wide range of advanced lung diseases. While the survival of lung transplant recipients continues to improve, infectious complications contribute substantially to morbidity and mortality following lung transplantation. The incidence of invasive fungal infections is variable, with a mean occurrence of $8.6 \%$. The majority of fungal infections in lung transplant recipients are caused Aspergillus and Candida species. This review provides an update in the current approaches for the diagnosis, management and prevention of fungal infections and the late complications that are associated.
\end{abstract}

Keywords: Fungal infection; lung transplantation; antifungal prophylaxis; invasive fungal infection

Submitted Dec 19, 2019. Accepted for publication Mar 09, 2020.

doi: $10.21037 /$ atm.2020.03.102

View this article at: http://dx.doi.org/10.21037/atm.2020.03.102

\section{Introduction}

Lung transplants are used to extend the lifespan and improve the quality of life of people suffering from severe or advanced chronic lung conditions. However, improving the long-term survival of this patient population remains a challenge. The first year following lung transplant surgery poses the highest risk of complications, such as rejection and infection. While the use of immunosuppressive agents reduces the incidence of rejection in the transplanted lung, this unfortunately also increases the risk of opportunistic infections (1) including invasive fungal disease (IFD). Fungal infections remain as one of the main causes of morbidity and mortality in this group of patients, and they are associated with a higher mortality compared with bacterial and viral infections (2).

According to the transplant associated infection surveillance network (TRANSNET), 8.6\% of lung transplant recipients (LTR's) develop invasive fungal infections (IFI's) during the first year after lung transplantation (3). However, reports across in multiple clinical trials show that this incidence rate is somewhat variable.

\section{Risk factors}

The risk of developing an invasive fungal infection varies depending on multiple factors; including the degree of host immunosuppression required to prevent rejection, and local defense mechanisms present in the host. Reduction of these host defense mechanisms following surgery-such as loss of lymphatics, denervating injury, reduced coughing and mucociliary clearance-predispose the patient to a higher risk of IFI's.

Yet another relevant risk factor in LTR's is the constant contact with the external environment, allowing pathogens direct access into the allograft. The majority of non-Candida fungal infections are acquired either through inhalation, or through pre-transplant colonization. Due to the ubiquitous nature of Aspergillus spp. spores in the air, all humans are 
Table 1 Risk factors for development of IFI's in lung transplant recipients $(6,8)$

Single lung transplant

Early airway ischemia

CMV infection

Rejection and augmented immunosuppression

Pre- or post-transplant Aspergillus colonization within a year after transplantation

Hypogammaglobulinemia (lgG $<400 \mathrm{mg} / \mathrm{dL}$ )

exposed to this fungus. In healthy individuals, epithelial cells within the respiratory tract, alveolar macrophages, and neutrophils are primarily responsible for eliminating the conidia and hyphae of Aspergillus spp. However, in immunocompromised individuals such as LTR's these functions tend to be impaired (4). Increased environmental exposure is commonly associated with activities such as farming, gardening and construction (5).

In the case of invasive aspergillosis (IA) specifically, the significant risk factors are single lung transplant (HR, 1.84; 95\% CI, 1.09-3.10; $\mathrm{P}=0.02$ ) and colonization with Aspergillus at 1-year post-transplantation (HR, 2.11; $95 \%$ CI, 1.28-3.49; $\mathrm{P}=0.003)$ (6).

In LTR's with cystic fibrosis, pre-transplant colonization with Aspergillus spp. has been reported to be as high as $70 \%$. Additionally, these patients have a 4-fold higher risk of IA (7). The risk factors for invasive fungal infection in LTR's are presented in Table 1.

\section{Clinical manifestations}

The most common pathogens that cause IFI's after solid organ transplantation (SOT) is invasive candidiasis (53\%) followed by IA (19\%) and cryptococcosis (8\%) (3).

Yet in LTR's, the most common IFI's are Aspergillus spp. (with $44-63 \%$ of these being caused by Aspergillus fumigatus), Candida spp. (with $23 \%$ being caused by Candida albicans) and other molds, such as Scedosporium spp. (20\%). Less commonly, other pathogens such as Cryptococcus neoformans, members of the Mucorales group, and other endemic mycoses can cause IFI in a small proportion of cases (9-11). This difference could be explained by multiple factors, such as patient exposure-because all humans are continuously exposed to Aspergillus spp., patient population, prior colonization, and center-dependent practices (including the use of antifungal prophylaxis).

Candidemia tends to occur within the first month posttransplant. It has been associated with significant morbidity and mortality. Some of the risk factors that has been associated with invasive candidemia are high dose steroids, immunomodulators, long term catheters, hospitalization prior to transplant, rejection, need for an open chest following the transplant procedure, and ECMO support post-transplant (12).

Aspergillus infection tend to occur within a mean time of 3-12 months after transplantation. The spectrum of infection caused by Aspergillus species is diverse. While IA occurs in $3-15 \%$ of the patients, $58 \%$ of these infections are tracheobronchitis or bronchial anastomotic infections, $32 \%$ invasive pulmonary aspergillosis (IPA) and $22 \%$ disseminated infections with extrapulmonary involvement (13).

In addition to these well-described pathogens, rare molds have emerged in the past few years including Rbizopus, Mucor, Rhizomucor, Cunninghamella, Scedosporium, Fusarium, Paecilomyces, Scopulariopsis, Acremonium, Trichoderma and others. These account for approximately $27 \%$ of mold infections, are more likely to be disseminated, and are associated with poorer outcomes $(14,15)$. These fungi are typically found within diverse environmental sources such as soil, water, vegetation and sewage.

\section{Anastomotic fungal infections}

Tracheobronchitis or anastomotic fungal infections typically are caused by Candida spp. and Aspergillus spp., and occur within the first 3 months after transplantation. They can be asymptomatic, or detectable only through either surveillance bronchoscopy or as a change in spirometry. Some patients may complain of noisy breathing, or being unable to cough up secretions. In bronchoscopy, these endobronchial lesions can be observed as erythema, ulcers, and necrotizing pseudomembrane formation. In some cases, patients can present with fever, cough, wheezing and/or hemoptysis. Bilateral LTR's in some previous studies have documented a higher incidence of bronchial anastomotic infections (16). Unfortunately, the mortality rate in LTR's with bronchial anastomotic Aspergillus infection is around $23 \%$ (17).

\section{Invasive fungal pneumonias}

Fungal pneumonias in LTR's generally occur later than 
Table 2 Fungal pathogens in lung transplant recipients $(14,18,19)$

\begin{tabular}{|c|c|}
\hline Pathogen & Usual clinical manifestation \\
\hline Fusarium spp. & Skin and soft tissue infection; pulmonary disease; extrapulmonary disease \\
\hline Scedosporium spp. & Pulmonary disease; disseminated disease \\
\hline Candida spp. & Anastomotic fungal infection; Candidemia \\
\hline $\begin{array}{l}\text { Dematiaceous molds: Exophiala, Alternaria, Curvularia, } \\
\text { Dactylaria, Cladophialophora and others }\end{array}$ & Skin and soft tissue infection; brain abscess \\
\hline $\begin{array}{l}\text { Endemic mycosis: Blastomycosis; Coccidioidomycosis; } \\
\text { Histoplasmosis }\end{array}$ & Fever unknown origin; pulmonary disease; disseminated disease \\
\hline
\end{tabular}

tracheobronchial aspergillosis. They are suspected based on changes in chest imaging such as new infiltrates, nodules, or micronodules. Some patients can be asymptomatic and only present with changes in spirometry, but the most common symptoms include fever, dry cough, dyspnea, and hemoptysis. The most common pathogen causing invasive fungal pneumonia is Aspergillus spp. In LTR's with IA, the mortality rate rises to $80 \%$ (17). Many of these infections can be acquired from the environment by inhalation, or alternatively can be a re-activation of an existing focus. In single-LTR's, IA was documented in the native lung, suggesting that the native lung may be the source for Aspergillus (18).

\section{Extrapulmonary disease}

In LTR's the most common site of infection is the lungs and airways. Other extrapulmonary disease include sinuses, orbits and central nervous system. Rarer sites of infection that have been documented include osteomyelitis, thoracic wound infection, pericarditis, endophthalmitis and retroperitoneal abscesses. In a 2003 review that included a cohort of 78 LTR's with aspergillosis, disseminated infection occurred in $10 \%$ of patients (18).

Table 2 presents the common fungal pathogens in lung transplantation and their usual clinical manifestations.

\section{Diagnosis}

The diagnosis of fungal infections in LTR's represents a clinical challenge due to a lack of precise diagnostic tools that can distinguish between invasive disease and colonization. While innovative tests using new diagnostic markers are being developed, an alternative strategy to increase the sensitivity and specificity of available assays may lie in the combination of currently used markers.

Many transplant centers worldwide perform routine bronchoscopies on LTR's following transplant, primarily in order to assess potential allograft rejection. It is also common to take microbiologic samples during these bronchoscopies. While fungal microorganisms are often found, it is nevertheless difficult to establish whether these cultures represent a fungal colonization, or an invasive infection.

The International Society for Heart and Lung Transplantation (ISHLT) defines fungal colonization as the presence of fungus in the respiratory secretions [sputum or bronchoalveolar lavage (BAL)] detected by culture, polymerase chain reaction (PCR) or biomarker [galactomannan (GM)/cryptococcal antigen] in the absence of symptoms, radiologic, and endobronchial changes.

IFD is defined as the presence of fungus in the respiratory secretions (sputum or BAL) detected by the culture, PCR or biomarker (GM/cryptococcal antigen) in the presence of symptoms, radiologic and endobronchial changes, or presence of histologic changes consistent with the fungal invasion of the tissue (4).

In LTR's, lung biopsy can be useful to prove the diagnosis; in the case of IPA a biopsy demonstrating tissue invasion is needed.

\section{Microbiology}

The gold standard of diagnosis for invasive fungal infection 
continues to be mycological culture and microscopic examination of the infected tissue. In LTR's, respiratory samples such as BAL and/or trans-bronchial lung biopsy are helpful in providing the diagnosis. However, in respiratory samples the sensitivity of fungal cultures tends to be low, ranging between $50-70 \%$ of all cases (20).

\section{Fungal biomarkers}

Due to the limitations of conventional culture methods, new diagnostic tools have been developed such as GM, $\beta$-D-glucan and PCR. Despite these developments, at the date reporting their utility in LTR patients is limited.

Aspergillus GM enzyme immunoassays detect polysaccharides that are present in the cell wall of Aspergillus species (21). A limitation of this method, however, is that other filamentous fungi can have GM present in their cell wall, possibly causing cross reactivity with this test in cases of histoplasmosis, fusariosis and talaromycosis. False positive GM has been documented in $20 \%$ of LTR's (22).

Serum GM should not be used in LTR's for the diagnosis of IA due to its low sensitivity ( 30\%) (23) in this population. Conversely, BAL GM in LTR's has a sensitivity of $60 \%$ and specificity of $98 \%$. Additionally, BAL GM has been shown to have a higher sensitivity, if a threshold value of $>1$ is used for determining positivity (22-25).

$\beta$-D-glucan is a component of the cell wall of most fungi, and therefore this test can have cross reactivity with other fungi (Candida or Pneumocystis). False-positive results have also been reported with beta-lactam antimicrobials and immunoglobulins. In LTR's the sensitivity has been reported $71-80 \%$ and specificity of $59-70 \%$ (26), therefore serum $\beta$-D-glucan is currently not recommended for the diagnosis of IFI's in LTR's.

Multiple DNA detection assays including PCR have been developed in the past decade for the diagnosis of IA. While the use of PCR is not included in the ISHLT definition of IFD, a 2011 report of BAL pan-Aspergillus PCR in LTR's had a sensitivity of $100 \%$ and specificity of $88 \%$ (27) with the limitation that it cannot differentiate between colonization and infection.

Other new diagnostic tools are under development, standardization, or validation. Such tools include urinary antigen Aspergillus detection, volatile organic compounds, lateral flow device using monoclonal antibodies, and other non-specific biomarkers such as Pentraxin-related protein (PTX3) and cytokines.

\section{Imaging}

Fungal infections present typical radiological findings in CT such as pulmonary nodules, air crescent sign, cavitation, and halo sign. Unfortunately, these CT findings have only been validated in high-risk, neutropenic, and bone marrow recipients. In LTR's, these typical findings are not as useful.

In one study the predominant CT finding in LTR's with IPA was bilateral bronchial wall thickening and centrilobular opacities with a tree-in-bud pattern. Ground-glass opacities and/or bilateral areas of consolidation were also common findings, and pulmonary nodules with halo sign were found in only $13 \%$ of patients (28).

New promising radiological techniques are being developed such as antibody-guided PET/MRI for the molecular imaging of fungal infections in vivo.

\section{Treatment}

Early initiation of antifungal therapy is critical for the treatment of IFI in LTR's. As is reducing prescribed immunosuppression whenever possible.

\section{Pulmonary aspergillosis}

Currently voriconazole is the drug of choice for treatment of IA. This recommendation is endorsed by the Infectious Diseases Society of America (IDSA) (29) and also by the American Society of Transplantation (AST) (30).

This recommendation is based in the findings of a randomized controlled trial of 144 patients (of which $6.2 \%$ were SOT recipients). Voriconazole was compared to amphotericin $\mathrm{B}$, and had a successful treatment outcome after 12 weeks of treatment of $52.8 \%$ vs. 31.6\% respectively. Additionally, it was associated with improved survival (70.8\% in the voriconazole group and $57.9 \%$ in the amphotericin group) (31). An important consideration when giving voriconazole (or other azoles) to SOT recipients are the significant interactions with tacrolimus, cyclosporine and sirolimus.

Since voriconazole can increase the levels of these immunosuppressants, it is recommended that serum trough concentrations are monitored 5-7 days into therapy. The target voriconazole trough level should be $>1$ to $<5.5 \mathrm{mcg} / \mathrm{mL}$, and should be rechecked every $1-2$ weeks. In cases of uncertainty regarding trough concentrations, it is recommended to repeat the levels every $3-5$ days (30).

Voriconazole has multiple additional adverse effects 
such as transient visual disturbances (blurriness, color changes), it can cause hepatoxicity mainly due to increased transaminases $(13 \%)$, rash $(6 \%)$, hallucination $(4.3 \%)$, QT prolongation, and enhanced risk of squamous cell carcinoma (32).

Another therapeutic option is isavuconazole. Using allcause mortality through day 42 as the primary endpoint (19\% vs. $20 \%$, respectively) it has been identified as non-inferior to voriconazole in invasive mold infections caused by Aspergillus and other filamentous fungi (33). In patients with liver insufficiency, azoles should be avoided, and instead liposomal amphotericin B is usually the first therapeutic option. Posaconazole is mainly used in cases of refractory or infection which is intolerant to other first-line antifungal agents (34).

\section{Other fungal infections}

In addition to Aspergillus spp., there are other important causes of fungal infection in LTR's, including Candida spp., Cryptococcus spp., Fusarium spp., Scedosporium spp., and dematiaceous molds. Each of these infections are treated differently due to their variable clinical presentation in LTR's.

Candida infection usually manifests as candidemia in the first month following lung transplantation. While uncommon, it is associated with high mortality (54.5\%). Risk factors for this invasive infection include high dose steroids, immunomodulators, long term catheters, rejection, need for open chest, and ECMO support post-transplant (12). In addition to candidemia other manifestations include invasive disease, pleural space infections, and local anastomotic site infections (35). The treatment of invasive candidiasis in LTR's is similar to the treatment of other patient populations; and is based on the 2016 and 2019 guidelines published by IDSA $(36,37)$. Echinocandins are recommended as initial therapy, with transition to azoles if the patient is clinically stable and the identified organism is susceptible.

Cryptococcosis is the third most common invasive fungal infection in SOT recipients. It is typically a lateoccurring infection, with the median time of 16-21 months post-transplant. It is primarily considered to represent reactivation of quiescent infection, however acquisition of primary infection, and transmission from donor organs and tissue grafts have also been described (38). There have been no randomized controlled trials of an antifungal therapy for cryptococcosis in SOT recipients. Thus, the current treatment recommendations are extrapolated from the $\mathrm{HIV}$-infected patients, and are consisted with the revised guidelines from the IDSA (39).

The incidence of infections by other filamentous fungi in transplant recipients has increased in recent years, predominately caused by Mucorales (mucormycosis or zygomycosis). Nonetheless, infections caused by Fusarium spp. and Scedosporium spp. are also increasing (38). Currently there are no specific recommendations for the management of these infections in LTR's, and the same management is extrapolated from other immunocompromised patient populations. Mucorales are resistant to many antifungal agents, and high-dose liposomal amphotericin is considered the first-line therapy (40). Because of the rapid spread of mucormycosis, antifungal therapy should be initiated immediately once the disease is suspected.

\section{Prevention}

Fungi are difficult to avoid because they are ubiquitous within the environment, and can be found either indoors or outdoors in soil, plants and vegetation. Certain occupations and living circumstances can put patients at higher risk, such as construction sites, farming operations, sandblasting, and working with air conditioning filters or on flooded sites.

Some of the recommendations that we can provide patients is to try to avoid areas with a lot of dust like construction or excavation sites, try to stay inside during dust storms, avoid contact with bird and bat droppings, wear shoes, gloves, long pants and long-sleeved shirt when doing outdoor activities like gardening, yard work or visiting wooded areas (41).

\section{Prophylaxis}

Currently, there are three strategies: universal prophylaxis, targeted prophylaxis, and preemptive therapy. According to ISHLT definitions (21):

* Universal prophylaxis refers to an antifungal medication started in the postoperative period in all patients, before any post-transplant isolation of a fungal pathogen.

* Targeted prophylaxis refers to an antifungal medication started in the post-transplant period before any fungal pathogen is isolated or serological marker of fungus is positive and is prescribed only to patients deemed at higher risk for IFD (e.g., cystic fibrosis patients and those with pre-transplant 
Table 3 Preventive strategies with antifungals in lung transplant recipients (20,30,46-48)

\begin{tabular}{llll}
\hline Strategy & Recommendation & Prophylaxis \\
\hline $\begin{array}{l}\text { Universal } \\
\text { prophylaxis }\end{array}$ & To all patients during the immediate post-transplant period & \\
$\begin{array}{lll}\text { Targeted } \\
\text { antifungal } \\
\text { prophylaxis }\end{array}$ & $\begin{array}{l}\text { In patients with any of the following risk factors: single lung transplant, early airway ischemia, } \\
\text { alemtuzumab or anti-thymocyte globulin, positive intraoperative Aspergillus culture in CF }\end{array}$ & Nebulized L-Amb; \\
& $\begin{array}{l}\text { patient, hypogammaglobulinemia, CMV infection; and that will be followed with BAL cultures } \\
\text { and BAL galactomannan }\end{array}$ & $\begin{array}{l}\text { posaconazole; } \\
\text { isavuconazole; usually for }\end{array}$ \\
& $\begin{array}{l}\text { Administration of antifungal agents for molds isolated during surveillance post-transplant } \\
\text { Preemptive }\end{array}$ & Depends on the mold \\
therapy & bronchoscopy without evidence of invasive disease & isolated & \\
\hline
\end{tabular}

fungal colonization, infection or augmented immunosuppression).

* Preemptive antifungal therapy refers to an antifungal medication started post-transplant isolation of a fungal pathogen or serologic marker of fungus, in the absence of any evidence for invasive fungal infection.

In LTR's the ideal strategy has not yet been delineated, due to the lack of randomized controlled studies. In a metaanalysis of universal prophylaxis vs. no antifungal prophylaxis in LTR's, 19 of 235 (8.1\%) and 28 of 196 (14.3\%) developed IA in the universal prophylaxis and no prophylaxis arms respectively (RR: 0.36; 95\% CI, 0.05-2.62). No significant reduction in IA or Aspergillus colonization with universal anti-aspergillus prophylaxis was found (42).

Another meta-analysis conducted in 2016 found that universal antifungal prophylaxis reduces the incidence of IA after lung transplantation (43). However, limitations of this meta-analysis included the fact that the included studies were quite heterogeneous, with some also not using multivariate adjustment in their analysis.

The use of universal prophylaxis has several identified disadvantages. Late cases of IA have been reported even in patients prescribed prophylaxis for a month post-transplant (18). Additionally, the use of azoles has several limitations, mainly in regards to the potential side effects (hepatotoxicity, neurotoxicity, QT prolongation), drug interactions, and emerging resistance (44). Furthermore, breakthrough IFI can occur despite antifungal prophylaxis (45).

The current recommendations of the AST Aspergillus guidelines (30) suggest that either universal prophylaxis or preemptive therapy can be employed as a strategy to prevent IA in LTR's. Table 3 presents the current recommendation of antifungal prophylaxis in LTR's.

\section{Complications}

\section{Invasive fungal infections and mortality}

Post-transplant survival has improved over time, despite considerable changes in patient characteristics and severity at the time of transplant. Currently, IFD is the second most common cause of mortality in LTR's, and is significantly associated with higher all-cause mortality after lung transplantation (HR, 2.70; 95\% CI: 1.23-5.79; $\mathrm{P}=0.0146$ ) (9).

Aspergillus infection in LTR's has been associated with a reduction in the 5-year survival rate of this patient group (49). This increased mortality is closely associated with the development of IPA, with $58 \%$ mortality after 2-year. Whereas colonization on the other hand was not associated with early increased mortality, but was associated with increased mortality after 5-year compared to non-infected patients (50). A previous review of Aspergillus infections in LTR's noted that late onset development of Aspergillus was associated with higher mortality than early onset disease (57\% vs. $28 \%, \mathrm{P}=0.045)$ (18).

Scedosporium species infection are the second most common cause of invasive mold infections overall. In one study, the mortality rate among transplant recipients (not exclusively LTR's) with scedosporiosis was 54\% (31 of 57), $77.8 \%$ for patients with $S$. prolificans infection, and $54.5 \%$ for patients with $S$. apiospermum infection. Another relevant finding was that the presence of disseminated infection $(\mathrm{OR}$, 0.20; $\mathrm{P}=0.03$ ) predicted lower survival (19).

Cryptococcosis is the third most common invasive fungal infection in LTR patients, and is also associated with a significant risk of dissemination and mortality (51). This disease typically occurs with reactivation of a latent focus of infection, and reported mortality ranges from 14-19.6\% $(52,53)$. Most cases of cryptococcosis occur later in the posttransplant period. 
Table 4 Complications after fungal invasive infections in lung transplant recipients

\begin{tabular}{lll}
\hline Complications & Mortality & Association with clad \\
\hline Invasive aspergillosis & $58 \%$ after 2-year post-lung transplant (50) & Reported (55-57) \\
Scedosporiosis & In SOT was 54\% (31 of 57) (47); 77.8\% S. prolificans infection; & Reported (58) \\
Cryptococcosis & 54.5\% S. apiospermum infection & \\
Candidiasis & In SOT population ranges from 14-19.6\% (52,53) & \\
Fusariosis & In SOT population 54\% (6 of 11) (12) & \\
\hline
\end{tabular}

In a case series of LTR's with fusariosis it was found that lung involvement occurred in all patients with disseminated disease, and that it was associated with a poor outcome. The mortality rate in this group of patients was high (67\%) (54). Table 4 further outlines the complications after fungal invasive infections in lung transplant recipients.

\section{Chronic lung allograft dysfunction}

Chronic lung allograft dysfunction (CLAD) remains a major hurdle limiting long-term survival post lung transplantation (59). CLAD is defined as substantial and persistent decline $(>20 \%)$ in measured $\mathrm{FEV}_{1}$ value from the reference (baseline) value. The baseline value is computed as the mean of the best 2 post-operative $\mathrm{FEV}_{1}$ measurements (taken $>3$ weeks apart) (60).

The phenotypes of CLAD have been clearly defined as bronchiolitis obliterans syndrome (BOS), restrictive allograft syndrome (RAS), mixed and undefined.

Infection is a recognized risk factor for the development of CLAD (61), and both acute infection and chronic lung allograft colonization with microorganisms have an increased risk of CLAD. Infection can lead to acute rejection through direct injury to the airway epithelial cells, or indirect injury through propagation of inflammatory responses. These mechanisms can increase the expression of alloantigens on the graft surface. Thus, providing alloimmune targets for pre-formed memory cells, which can activate and differentiate to new secondary effector cells that directly damage the graft tissue $(55,62)$. Chronic rejection can occur when these secondary differentiated effector T-cells persist and develop into long-life memory T-cells. These become very specific for alloantigens, and are able to recognize major histocompatibility complex molecules on the graft surface $(55,56)$.

Multiple bacterial and viral infections have been associated with the development of CLAD such as $S$. aureus, P. aeruginosa, and CMV (63). However fungal colonization and infection of the lung allograft is associated with the development of, and mortality due to $\operatorname{CLAD}(55,57)$.

One of the earliest studies on the effect of fungal infections and the development of BOS reported that fungal pneumonias in the first 100 days post-transplant were associated with potential BOS with a hazard ratio of 2.1 (95\% CI, 1.1-4.0). If these fungal infections occurred in the late post-operative period, they were also associated with BOS with a HR 1.5 (95\% CI, 1.1-1.9) (55).

Fungal infection alone is not solely responsible for the development of CLAD. Fungal colonization has also been associated with its development. Fungal colonization after lung transplantation is very common, especially due to Aspergillus spp. Allograft airway colonization by Aspergillus leads to persistent airway inflammation that eventually contributes to the development of BOS.

Out of all fungal infections, Aspergillus spp. is the perpetrating organism that has been studied most extensively in association with CLAD. Weigt et al., in a 2009 retrospective study found that Aspergillus colonization strongly preceded the development of potential CLAD (by a median of 184 days and stage 1 or greater by a median of 261 days) and CLAD-related mortality in the Cox regression analyses. Aspergillus colonization was also identified as a distinct risk factor for CLAD, independent of acute rejection (57).

In a follow-up validation study of LTR's across two centers, colonization with small conidia Aspergillus $(<3.5 \mathrm{mcm})$ was associated with an increased risk of developing CLAD (HR, 1.44; 95\% CI, 1.14-1.82, $\mathrm{P}=0.002)$ (64) small conidial species included $A$. fumigatus, $A$. nidulans, A. terrus, and $A$. flavipes. Greater conidia species included A. niger, A. flavus, A. ustus, and A. clavatus. Prospective strategies to prevent Aspergillus colonization of the graft is warranted, with the goal of preventing CLAD and mortality after CLAD.

Some other fungi have been associated with CLAD. In a retrospective review, Scedosporium spp. colonization or 
infection was associated with increased risk for BOS (HR, 6.67; 95\% CI, 1.83-24.29; $\mathrm{P}=0.004)$ and death (HR, 5.37; 95\% CI, 2.29-12.59, $\mathrm{P}<0.0001$ ) (58). More additional studies need to better elucidate the role of non-Aspergillus molds in CLAD.

\section{Conclusions}

Fungal infections continue to be a significant cause of life-threatening infection in LTR's. The diagnosis of fungal infections should be a combination of clinical and radiological factors, as well as invasive and non-invasive tests such as fungal staining, culture, BAL GM, and biopsies. Optimal management includes early diagnosis and early initiation of antifungal therapy. While the most effective prophylaxis and preventive strategy has not yet been determined, it should probably be individualized depending on specific patient factors. Fungal infection and colonization are associated with the development of CLAD and mortality.

\section{Acknowledgments}

Funding: None.

\section{Footnote}

Provenance and Peer Review: This article was commissioned by the Guest Editors (Masaaki Sato and Dong Tian) for the series "Strategies to Achieve Long-Term Success of Lung Transplantation" published in Annals of Translational Medicine. The article was sent for external peer review organized by the Guest Editors and the editorial office.

Conflicts of Interest: The series "Strategies to Achieve LongTerm Success of Lung Transplantation" was commissioned by the editorial office without any funding or sponsorship. The authors have no conflicts of interest to declare.

Ethical Statement: The authors are accountable for all aspects of the work in ensuring that questions related to the accuracy or integrity of any part of the work are appropriately investigated or resolved.

Open Access Statement: This is an Open Access article distributed in accordance with the Creative Commons Attribution-NonCommercial-NoDerivs 4.0 International License (CC BY-NC-ND 4.0), which permits the non- commercial replication and distribution of the article with the strict proviso that no changes or edits are made and the original work is properly cited (including links to both the formal publication through the relevant DOI and the license). See: https://creativecommons.org/licenses/by-nc-nd/4.0/.

\section{References}

1. Fishman JA, Rubin RH. Infection in organ-transplant recipients. N Engl J Med 1998;338:1741-51.

2. Mattner F, Fischer S, Weissbrodt H, et al. Post-operative nosocomial infections after lung and heart transplantation. J Heart Lung Transplant 2007;26:241-9.

3. Pappas PG, Alexander BD, Andes DR, et al. Invasive fungal infections among organ transplant recipients: results of the Transplant-Associated Infection Surveillance Network (TRANSNET). Clin Infect Dis 2010;50:1101-11.

4. Husain S, Sole A, Alexander BD, et al. The 2015 International Society for Heart and Lung Transplantation Guidelines for the management of fungal infections in mechanical circulatory support and cardiothoracic organ transplant recipients: Executive summary. J Heart Lung Transplant 2016;35:261-82.

5. Raviv Y, Kramer MR, Amital A, et al. Outbreak of aspergillosis infections among lung transplant recipients. Transpl Int 2007;20:135-40.

6. Aguilar CA, Hamandi B, Fegbeutel C, et al. Clinical Risk Factors for Invasive Aspergillosis in Lung Transplant Recipients: Results of an International Cohort Study. J Heart Lung Transplant 2018;37:1226.

7. Luong ML, Chaparro C, Stephenson A, et al. Pretransplant Aspergillus colonization of cystic fibrosis patients and the incidence of post-lung transplant invasive aspergillosis. Transplantation 2014;97:351-7.

8. Camargo JF, Husain S. Immune correlates of protection in human invasive aspergillosis. Clin Infect Dis 2014;59:569-77.

9. Arthurs SK, Eid AJ, Deziel PJ, et al. The impact of invasive fungal diseases on survival after lung transplantation. Clin Transplant 2010;24:341-8.

10. Frost AE. Role of infections, pathogenesis, and management in lung transplantation. Transplant Proc 1999;31:175-7.

11. Avery RK. Infections after lung transplantation. Semin Respir Crit Care Med 2006;27:544-51.

12. Gadre SK, Koval C, Budev M. Candida Blood Stream Infections Post Lung Transplant. J Heart Lung Transplant 2017;36:S241.

13. Singh N, Paterson DL. Aspergillus infections in transplant 
recipients. Clin Microbiol Rev 2005;18:44-69.

14. Solé A, Salavert $M$. Fungal infections after lung transplantation. Curr Opin Pulm Med 2009;15:243-53.

15. Shoham $S$. Emerging fungal infections in solid organ transplant recipients. Infect Dis Clin North Am 2013;27:305-16.

16. Hadjiliadis D, Howell DN, Davis RD, et al. Anastomotic infections in lung transplant recipients. Ann Transplant 2000;5:13-9.

17. Husain S, Paterson DL, Studer S, et al. Voriconazole prophylaxis in lung transplant recipients. Am J Transplant 2006;6:3008-16.

18. Singh N, Husain S. Aspergillus infections after lung transplantation: clinical differences in type of transplant and implications for management. J Heart Lung Transplant 2003;22:258-66.

19. Husain S, Munoz P, Forrest G, et al. Infections due to Scedosporium apiospermum and Scedosporium prolificans in transplant recipients: clinical characteristics and impact of antifungal agent therapy on outcome. Clin Infect Dis 2005;40:89-99.

20. Geltner C, Lass-Flörl C. Invasive pulmonary Aspergillosis in organ transplants - Focus on lung transplants. Respir Investig 2016;54:76-84.

21. Herrera S, Husain S. Early diagnosis of fungal infections in lung transplant recipients, colonization versus invasive disease? Curr Opin Organ Transplant 2018;23:381-7.

22. Husain S, Kwak EJ, Obman A, et al. Prospective assessment of Platelia Aspergillus galactomannan antigen for the diagnosis of invasive aspergillosis in lung transplant recipients. Am J Transplant 2004;4:796-802.

23. Pfeiffer CD, Fine JP, Safdar N. Diagnosis of invasive aspergillosis using a galactomannan assay: a meta-analysis. Clin Infect Dis 2006;42:1417-27.

24. Zou M, Tang L, Zhao S, et al. Systematic review and metaanalysis of detecting galactomannan in bronchoalveolar lavage fluid for diagnosing invasive aspergillosis. PLoS One 2012;7:e43347.

25. Husain S, Paterson DL, Studer SM, et al. Aspergillus galactomannan antigen in the bronchoalveolar lavage fluid for the diagnosis of invasive aspergillosis in lung transplant recipients. Transplantation 2007;83:1330-6.

26. Bhaskaran A, Kabbani D, Singer LG, et al. $(1,3)$ beta-DGlucan in Bronchoalveolar Lavage of Lung Transplant Recipients for the Diagnosis of Invasive Pulmonary Aspergillosis. Med Mycol 2017;55:173-9.

27. Luong ML, Clancy CJ, Vadnerkar A, et al. Comparison of an Aspergillus real-time polymerase chain reaction assay with galactomannan testing of bronchoalvelolar lavage fluid for the diagnosis of invasive pulmonary aspergillosis in lung transplant recipients. Clin Infect Dis 2011;52:1218-26.

28. Gazzoni FF, Hochhegger B, Severo LC, et al. Highresolution computed tomographic findings of Aspergillus infection in lung transplant patients. Eur J Radiol 2014;83:79-83.

29. Patterson TF, Thompson GR 3rd, Denning DW, et al. Practice Guidelines for the Diagnosis and Management of Aspergillosis: 2016 Update by the Infectious Diseases Society of America. Clin Infect Dis 2016;63:e1-60.

30. Husain S, Camargo JF. Invasive Aspergillosis in solidorgan transplant recipients: Guidelines from the American Society of Transplantation Infectious Diseases Community of Practice. Clin Transplant 2019;33:e13544.

31. Herbrecht R, Denning DW, Patterson TF, et al. Voriconazole versus amphotericin $\mathrm{B}$ for primary therapy of invasive aspergillosis. N Engl J Med 2002;347:408-15.

32. Eiden C, Peyriere H, Cociglio M, et al. Adverse effects of voriconazole: analysis of the French Pharmacovigilance Database. Ann Pharmacother 2007;41:755-63.

33. Maertens JA, Raad, II, Marr KA, et al. Isavuconazole versus voriconazole for primary treatment of invasive mould disease caused by Aspergillus and other filamentous fungi (SECURE): a phase 3, randomised-controlled, noninferiority trial. Lancet 2016;387:760-9.

34. Hachem RY, Langston AA, Graybill JR, et al. Posaconazole as salvage treatment of invasive fungal infections in patients with underlying renal impairment. J Antimicrob Chemother 2008;62:1386-91.

35. Palmer SM, Perfect JR, Howell DN, et al. Candidal anastomotic infection in lung transplant recipients: successful treatment with a combination of systemic and inhaled antifungal agents. J Heart Lung Transplant 1998;17:1029-33.

36. Pappas PG, Kauffman CA, Andes DR, et al. Clinical Practice Guideline for the Management of Candidiasis: 2016 Update by the Infectious Diseases Society of America. Clin Infect Dis 2016;62:e1-50.

37. Aslam S, Rotstein C, Practice ASTIDCo. Candida infections in solid organ transplantation: Guidelines from the American Society of Transplantation Infectious Diseases Community of Practice. Clin Transplant 2019;33:e13623.

38. Gavaldà J, Meije Y, Fortun J, et al. Invasive fungal infections in solid organ transplant recipients. Clin Microbiol Infect 2014;20 Suppl 7:27-48.

39. Perfect JR, Dismukes WE, Dromer F, et al. Clinical practice guidelines for the management of cryptococcal 
disease: 2010 update by the infectious diseases society of america. Clin Infect Dis 2010;50:291-322.

40. Crabol Y, Lortholary O. Invasive mold infections in solid organ transplant recipients. Scientifica (Cairo) 2014;2014:821969.

41. Avery RK, Michaels MG. Strategies for safe living after solid organ transplantation. Am J Transplant 2013;13:304-10.

42. Bhaskaran A, Mumtaz K, Husain S. Anti-Aspergillus Prophylaxis in Lung Transplantation: A Systematic Review and Meta-analysis. Curr Infect Dis Rep 2013;15:514-25.

43. Pilarczyk K, Haake N, Heckmann J, et al. Is universal antifungal prophylaxis mandatory in adults after lung transplantation? A review and meta-analysis of observational studies. Clin Transplant 2016;30:1522-31.

44. Perlin DS, Rautemaa-Richardson R, Alastruey-Izquierdo A. The global problem of antifungal resistance: prevalence, mechanisms, and management. Lancet Infect Dis 2017;17:e383-92.

45. Baker AW, Maziarz EK, Arnold CJ, et al. Invasive Fungal Infection After Lung Transplantation: Epidemiology in the Setting of Antifungal Prophylaxis. Clin Infect Dis 2020;70:30-9.

46. Borro JM, Sole A, de la TM, et al. Efficiency and safety of inhaled amphotericin B lipid complex (abelcet) in the prophylaxis of invasive fungal infections following lung transplantation. Transplant Proc 2008;40:3090-3.

47. Neoh CF, Snell GI, Levvey B, et al. Preemptive treatment with voriconazole in lung transplant recipients. Transpl Infect Dis 2013;15:344-53.

48. Husain S, Bhaskaran A, Rotstein C, et al. A strategy for prevention of fungal infections in lung transplantation: Role of bronchoalveolar lavage fluid galactomannan and fungal culture. J Heart Lung Transplant 2018;37:886-94.

49. Solé A, Morant P, Salavert M, et al. Aspergillus infections in lung transplant recipients: risk factors and outcome. Clin Microbiol Infect 2005;11:359-65.

50. Iversen M, Burton CM, Vand S, et al. Aspergillus infection in lung transplant patients: incidence and prognosis. Eur J Clin Microbiol Infect Dis 2007;26:879-86.

51. Sohail A, Smibert OC, Snell G, et al. Cryptococcal infection in lung transplant recipients: A 5-year retrospective review at an Australian transplant center. Transpl Infect Dis 2018;20:e12976.

52. Wu G, Vilchez RA, Eidelman B, et al. Cryptococcal meningitis: an analysis among 5,521 consecutive organ transplant recipients. Transpl Infect Dis 2002;4:183-8.

53. Sun HY, Wagener MM, Singh N. Cryptococcosis in solid- organ, hematopoietic stem cell, and tissue transplant recipients: evidence-based evolving trends. Clin Infect Dis 2009;48:1566-76.

54. Carneiro HA, Coleman JJ, Restrepo A, et al. Fusarium infection in lung transplant patients: report of 6 cases and review of the literature. Medicine (Baltimore) 2011;90:69-80.

55. Valentine VG, Gupta MR, Walker JE Jr, et al. Effect of etiology and timing of respiratory tract infections on development of bronchiolitis obliterans syndrome. J Heart Lung Transplant 2009;28:163-9.

56. Cakała M, Olszewski WL. Memory response in allografting and bacterial infections in lymphoid and nonlymphoid tissues. Ann Transplant 2006;11:38-44.

57. Weigt SS, Elashoff RM, Huang C, et al. Aspergillus colonization of the lung allograft is a risk factor for bronchiolitis obliterans syndrome. Am J Transplant 2009;9:1903-11.

58. Kim J, Greenland J, Cohen S, et al. Scedosporium is a Risk Factor for Bronchiolitis Obliterans Syndrome in Lung Transplantation. ID Week; Oct 5.; San Franscisco, CA, USA: Infectious Diseases Society of America; 2013.

59. Gauthier JM, Hachem RR, Kreisel D. Update on Chronic Lung Allograft Dysfunction. Curr Transplant Rep 2016;3:185-91.

60. Verleden GM, Glanville AR, Lease ED, et al. Chronic lung allograft dysfunction: Definition, diagnostic criteria, and approaches to treatment- A consensus report from the Pulmonary Council of the ISHLT. J Heart Lung Transplant 2019;38:493-503.

61. Gregson AL. Infectious Triggers of Chronic Lung Allograft Dysfunction. Curr Infect Dis Rep 2016;18:21.

62. Crespo E, Lucia M, Cruzado JM, et al. Pre-Transplant Donor-Specific T-Cell Alloreactivity Is Strongly Associated with Early Acute Cellular Rejection in Kidney Transplant Recipients Not Receiving T-Cell Depleting Induction Therapy. PLoS One 2015;10:e0117618.

63. Belperio J, Palmer SM, Weigt SS. Host-Pathogen Interactions and Chronic Lung Allograft Dysfunction. Ann Am Thorac Soc 2017;14:S242-6.

64. Weigt SS, Copeland CAF, Derhovanessian A, et al. Colonization with small conidia Aspergillus species is associated with bronchiolitis obliterans syndrome: a twocenter validation study. Am J Transplant 2013;13:919-27.

Cite this article as: Villalobos APC, Husain S. Infection prophylaxis and management of fungal infections in lung transplant. Ann Transl Med 2020;8(6):414. doi: 10.21037/ atm.2020.03.102 